\title{
Influence of Microstructure on Materials Fatigue Life with Different MSZ Coverage Rates under Contact Loads
}

\author{
Deng Zhongmin ${ }^{1}$, Zhao Yibing ${ }^{2}$ and Hong Youshi ${ }^{2}$ \\ ${ }^{1}$ School of Space Technology, Beijing University of Aeronautics and Astronautics, Beijing 100083, \\ China \\ ${ }^{2}$ LNM, Institute of Mechanics, Chinese Academy of Sciences, Beijing 100080, China
}

Kevwords: Mechanical property, surface layer, laser treatment, MSZ coverage rate

Abstract. This paper investigates the effects of microstructure of materials with different coverage rates of melting-solidification zone (MSZ) due to laser treatment on surface stress amplitude and surface crack propagation under contact loading by using finite element method. The influence of MSZ rate on the contact fatigue life is then predicted. In the simulation, the difference of surface contour for different MSZ rates is taken into account. The results show that the mechanical properties of the surface with $20 \% \mathrm{MSZ}$ rate are relatively superior.

\section{Introduction}

The study of materials surface/interface mechanical properties is an important direction of solid mechanics in 21 st century [1]. The main factors, which affect materials surface mechanical property under contact loads are : applied load magnitude, profiles of contact pairs, friction coefficients, etc. These parameters will influence the contact stress, response of materials and therefore fatigue crack initiation. Lubricants affect friction condition, contact stresses, and rate and type of wear, and hence affect both crack initiation and propagation [2].

Laser texturing is a surface modification technology developed recently, and has good application foreground [3]. It can form designed distribution of remelt-pits with different MSZ coverage rates by adjusting parameters of laser treatment. The investigation on the effects of MSZ rate on material strength and toughness under contact loads has basic significance for improving techniques and applications of the materials surface treatment technology [4].

This paper analyzes the effects of MSZ rate on surface layer cyclic-stress amplitude and crack propagation under contact loads. It predicts the effect of MSZ coverage rate by laser treatment on the material strength and toughness under contact loads.

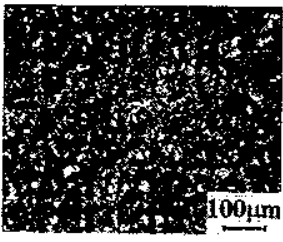

(a) MSZ rate $: 100 \%$

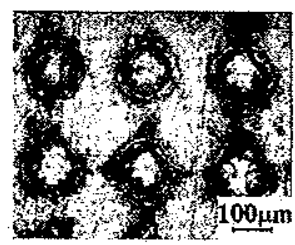

(c) MSZ rate $37.5 \%$

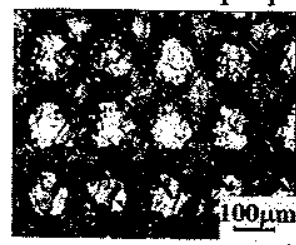

(b) MSZ rate: $78.5 \%$

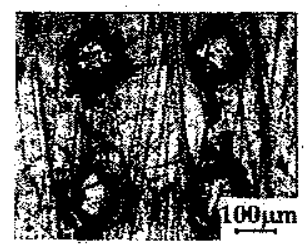

(d) MSZ rate $19.6 \%$

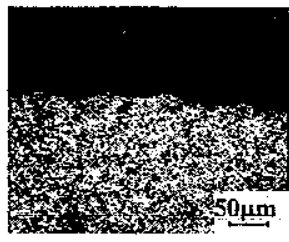

(e) Profile of $78.5 \%$

Fig.1 Microstructure of laser textured surface [5].

\section{Finite Element Model}

The arranged pits in materials surface are formed by laser treatment. The optical microscopy (OM) photos of four MSZ rates: $100 \%, 78.5 \%$, 
$37.5 \%, 19.6 \%$ are shown in Fig. 1 (a)-(d) respectively, and the profile of materials surface of MSZ rate $78.5 \%$ is displayed in Fig. 1 (e).

According to the ring/block wear experiments of the specimen with laser treatment, $2 \mathrm{D} \mathrm{FE}$

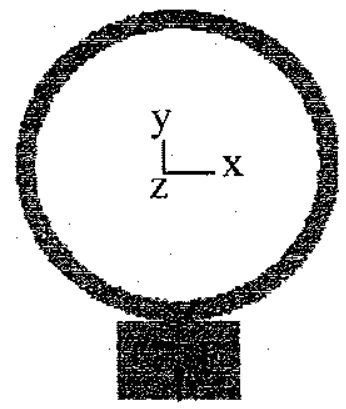

Fig.2 Ring/block wear scheme.

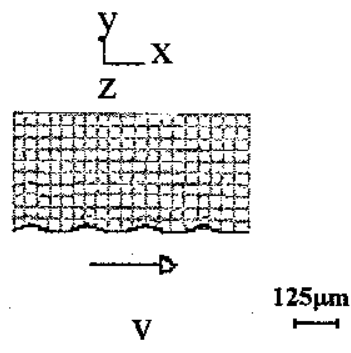

Fig. 3 Schematic of mesh of ring surface layer.

model is built. The difference of surface contour for laser treatment is considered. The corresponding curves have been used to simulate the materials contact surface. A ring contacts a block, and rotates around the fixed axis with $60 \mathrm{cir} / \mathrm{min}$. Both sides of the block are fixed, and load F is applied to the block lower surface (Fig.2) .

The calculated parameters are the same with the practical sample. Ring material is 18-8 type austenite stainless steel $(1 \mathrm{Cr} / 8 \mathrm{Ni} 9 \mathrm{Ti})$. The stress-strain curve of austenite stainless steel is get from the experiment: elastic modulus $E=210 \mathrm{GPa}$, Poisson ratio $v=0.29$ and yield strength $=243 \mathrm{MPa}$. Block material is $40 \mathrm{Cr}$, and materials parameters are $E=212 \mathrm{GPa}, v=0.28$ and yield strength $=$ $780 \mathrm{MPa}$.

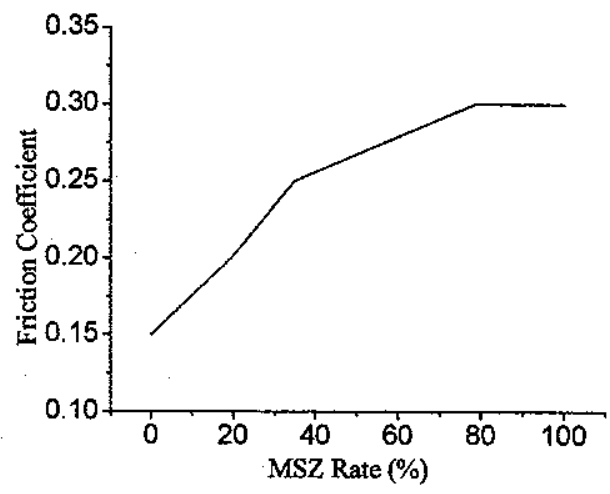

Fig. 4 Friction coefficients vs. MSZ rate.

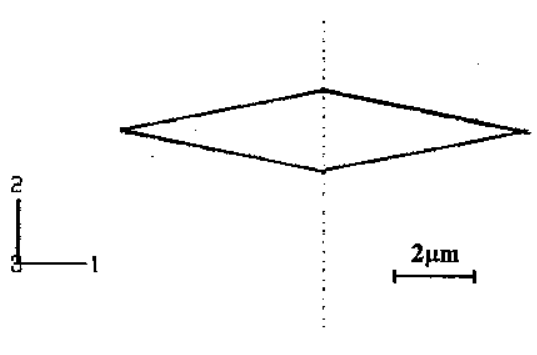

Fig.5 Figure of prefabricate crack.'

Some assumptions have been adopted in the calculation: 1) the increase of temperature by contact friction is not considered; and 2) the friction coefficients of all contact pairs are constant in contact processing. The numerical simulations are performed with the general purpose finite element code ABAQUS [6]. The ring surface treated by laser is defined as the main surface of contact pair, and the slave surface is block surface. Coulomb friction model is used in calculating.

The surface microstructural variables of contact pair (especially the shape, size of laser remelt pit) are considered in the FE mesh. For its symmetry, the FE model is modeled as a part of the ring, and its transient contact stress distributions are analyzed. Remelt pit is $70 \mu \mathrm{m}$ in radius, and the element 
length is $50 \mu \mathrm{m}$, plots by equal length. In this mesh case, the calculated results are stable. Block element length is $660 \mu \mathrm{m}$. All about 15000 elements are plotted, and Fig. 3 is the schematic of mesh of $1 \mathrm{~mm} \times 0.5 \mathrm{~mm}$ region of ring surface layer. In Fig. 3 , the lower surface is simulated as material laser remelt surface (contact main surface). Ring rotates around $z$ axes with velocity $v$.

Two cases, the same and different friction coefficients with different MSZ rates, are considered. In the 1 st case, the friction coefficient is 0.3 , and in the 2 nd case, the friction coefficient is obtained from Fig. 4.

For further considering the influence on material contact fatigue properties by different MSZ rates, the initiate crack is designed in the ring surface layer. The distance of the symmetric plane of crack to the surface of ring is $120 \mu \mathrm{m}$, the crack length $10 \mu \mathrm{m}$, width $2 \mu \mathrm{m}$, see Fig.5.

It plots about $2 \mu \mathrm{m}$ element length near the crack, and contact load is $20 \mathrm{~N}$ and friction coefficient equals to 0.3 .

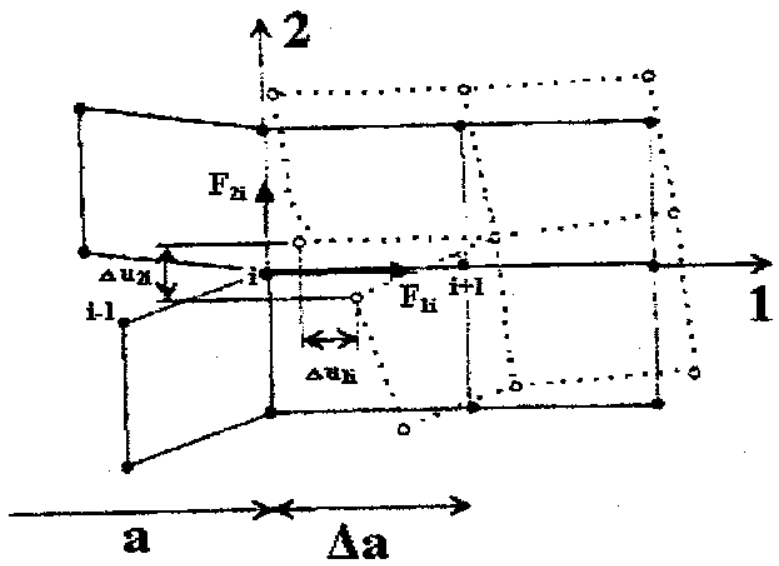

Fig.6 Crack-tip displacements and forces [7].

\section{Measures of Driving Forces on Crack}

The driving forces on crack are evaluated in two different ways - with crack-tip displacements and with energy release rate. The crack-tip displacements are measured at the crack face nodes pair next to the tip (Fig. 6). These displacements are divided into normal displacement to the crack plane, $\Delta u_{2 i}(a)$ the crack tip opening displacements CTOD, and displacements parallel to the crack plane, $\Delta u_{i i}(a)$ the crack tip shear displacements CTSD.

The energy release rate is estimated with the following approximated expression [7]:

$$
G=\frac{1}{\Delta a}\left[F_{1(i+1)}(a+\Delta a) \Delta u_{i j}(a+\Delta a)+F_{2(i+1)}(a+\Delta a) \Delta u_{2 i}(a+\Delta a)\right],
$$

where, $F_{j(i+1)}(a+\Delta a)$ are both nodal forces at the crack tip node, and crack tip openings at the node pair next to the tip, $\Delta u_{j i}(a+\Delta a)$, are evaluated for the same crack length, $a+\Delta a$.

The value of $\mathrm{G}$ expressed by Eq.1 is a sum of the energy release from displacements parallel $(\mathrm{j}=$ 1 ), and normal $(j=2)$, to the crack plane. The CTOD and the energy release rate have been used here to evaluate the driving forces.

\section{Results and Discussion}

In the $\log -\log$ coordinate system, the bias part of materials $\mathrm{S}-\mathrm{N}$ curves can be expressed : 


$$
\sigma^{m} N=C,
$$

where $m$ and $C$ are materials constants. Eq.2 can be written :

$$
N=\frac{C}{\sigma^{m}} \text {. }
$$

Eq. 3 shows that the material fatigue life will decrease as the contact stresses amplitude

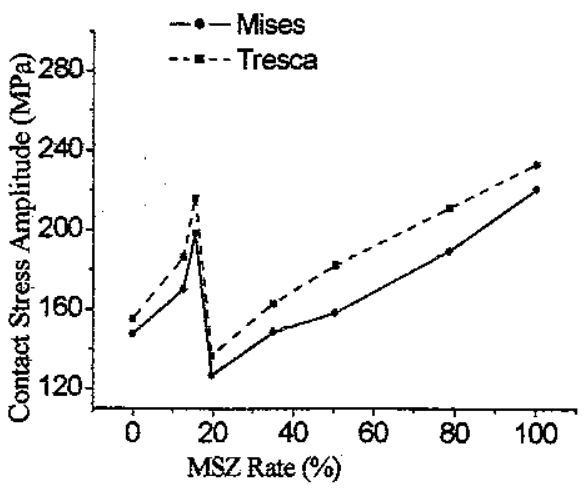

(a) The 1st case

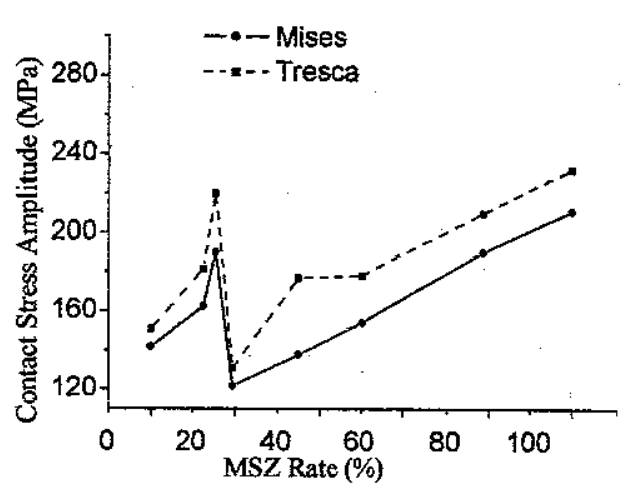

(b) The 2nd case

Fig . 7 Laser MSZ rate and contact stress amplitude.

increased.

Based on the FE analysis, Fig.7 shows Mises and Tresca equivalent stress amplitudes of the contact zone of material surface layer with different MSZ rates: (a) the 1st case, load $F$ equals $20 \mathrm{~N}$, and (b) the 2nd case, load $F$ equals $20 \mathrm{~N}$ too. Figure 8 illustrates the CTOD of the crack in the surface layer as a function of MSZ rates for the treated materials by laser. The wear experimental curve of loss weights is shown in Fig. 9, which presents that the loss weights of the material with laser treatment after worn for 8 hours.

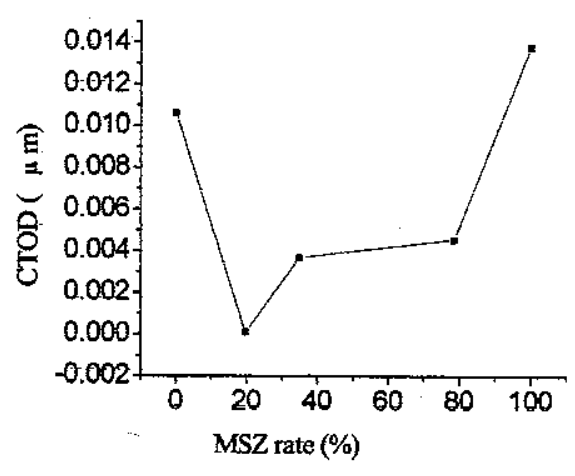

Fig. 8 CTOD vs. MSZ rate.

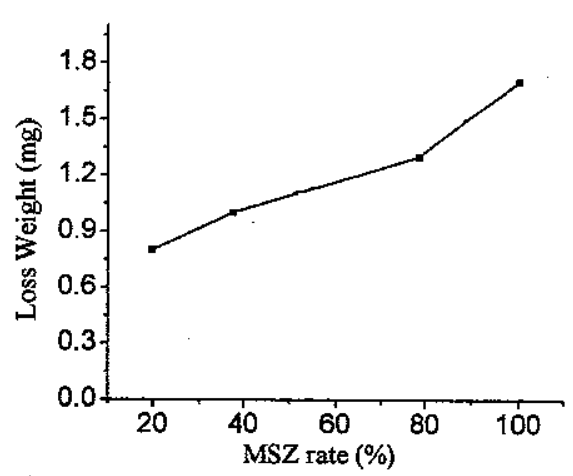

Fig. 9 Materials loss weights vs. MSZ rate. table:

The values of energy release rate with the different MSZ rates are shown in the following 
Table 1: The values of energy release rate G corresponding the MSZ rate

\begin{tabular}{|c|c|c|c|c|c|}
\hline MSZ rate(\%) & 0 & 19.6 & 35.5 & 78.5 & 100 \\
\hline $\mathrm{G}(\mathrm{N} / \mathrm{mm})$ & $3.09 \times 10^{-3}$ & $8.57 \times 10^{-4}$ & $3.12 \times 10^{-3}$ & $1.41 \times 10^{-2}$ & $8.82 \times 10^{-2}$ \\
\hline
\end{tabular}

According to Eq. 3, Fig.7 means that the contact fatigue life of the material is not proportional to the increase of the MSZ rate, the CTOD (Fig.8) and the energy release rate (Table 1), characterizing the cracking driving force is also not proportional to the increase of the MSZ rate. In Fig.7, while the MSZ rate equals to about $20 \%$, the Mises and Tresca contact stresses at material surface layer are the smallest for both cases. It predicts that its fatigue contact life with $20 \% \mathrm{MSZ}$ rate is higher than that of the other MSZ rates under the same rotate frequency and contact condition according to Eq. 3. Same trends are seen from Fig. 8 and Table 1. The CTOD and $\mathrm{G}$ are the smallest with $20 \%$ MSZ rate. It indicates that the fatigue resistance is the best in the MSZ rate.

Comparing Fig.7 (a) with (b), it exhibits that the friction coefficients have a little effect on contact stress amplitude (both Mises and Tresca) of material surface layer.

The wear experimental results in Fig.9 are the loss weights of the material with MSZ rate $100 \%$, $78.5 \%, 37.5 \%, 19.6 \%$ respectively (no experimental data of the material with MSZ rate $0 \%$ is obtained.), and show the agreement with numerical simulation. It is interesting that the push-pull fatigue experimental results of $1 \mathrm{Cr} 18 \mathrm{Ni} 9 \mathrm{Ti}$ [5] show the same trends with the numerical simulation of this paper.

\section{Conclusion}

The wear properties of the materials are different for varying MSZ rates and surface contour. The numerical simulation results show that the wear properties are not proportional to MSZ rate, and the wear properties will be better while the MSZ rate of materials nearly equal to $20 \%$. The numerical results agree with wear experiments of the materials.

\section{References}

[1] Z.M Zheng, H.Zhou, H.X.Zhang, K.Z Huang and Y.L.Bai, "Mechanics" , Sciences and developments at the beginning of 21 st century, Science publishing company, (1996), p.39 (In Chinese)

[2] J.W.Ringsberg, M.Loo-Morrey, B.L.Josefson, A.Kapoor and J.H.Beynon, International Journal of Fatigue, Vol. 22 (2000), p. 205

[3] V.V.Semark and N.B.Dahoter, Laser Surface Texture, Lasers in Surface Engineering, ASM International, (1998), p. 35

[4] S.W.Yu, Z.P.Duan and Y.S.Hong, Several problems of modern technique and mechanics. Modern mechanics and progress of science and technology, Aug.(1997), p. 85 (In Chinese)

[5] Y.B.Zhao, L.H.Peng and Y.S.Hong, Effects on fatigue properties of alloy steel by laser remelt, Mechanics 2000, 2000.8, Beijing (In Chinese)

[6] "ABAQUS/Explicit User Manual", Version 5.8

[7] A.Melander, International Journal of Fatigue, Vol. 19 (1997), p. 13. 